\title{
Correlações entre o processo de Definição do Processo Organizacional do Nível E do MPS.BR e as Características da Transparência de Software
}

\author{
Matheus Pedra Puime Feijoó ${ }^{1}$, Sabrina Santos Cruz de Oliveira ${ }^{1}$, Henrique Prado \\ de Sá Sousa ${ }^{1}$
}

${ }^{1}$ Departamento de Computação- Universidade Federal Rural do Rio de Janeiro (UFRRJ) Rodovia BR 465, Km 07, s/n - Zona Rural, Seropédica - RJ, 23890-000

\{feijoo,sabrina $\} @$ pet-si.ufrrj.br, \{hps.infotec $\} @$ gmail.com

Abstract. Quality is a crosscutting element both to the software development process and to the products and may be analyzed under different perspectives. The MPS.BR is a model that aims to improve quality in software processes. It was observed that these qualities are related to the characteristics of the Software Transparency model, demonstrating that the quality of Transparency is a driver of improvements in software products and processes. In this work the relations of the process of Organizational Process Definition, present in Level E of the MPS.BR, with the characteristics of Software Transparency, are investigated. Based on the identification of the qualitative relationships, some operational procedures are proposed to both instantiate the MPS.BR process and increase its Transparency degree.

Resumo. A qualidade é um elemento transversal tanto ao processo de desenvolvimento de software quanto ao produto, podendo ser analisado sob diversas perspectivas. O MPS.BR é um modelo que visa a melhoria dos processos de software em termos qualitativos. Observou-se que estas qualidades se relacionam às características do modelo de Transparência de Software, demonstrando que a qualidade de Transparência é um fator impulsionador de melhorias em processos e produtos de software. Neste trabalho são investigadas as relações do processo de Definição do Processo Organizacional, presente no Nivel E do MPS.BR, com as características de Transparência de Software. A partir da identificação das relações qualitativas, são propostas operacionalizações que visam tanto instanciar o processo MPS.BR quanto ampliar o seu grau Transparência.

\section{Introdução}

Os modelos de maturidade de processo desempenham um importante papel na busca do aperfeiçoamento de processos de construção de software dentro das organizações que a utilizam. Isso ocorre porque os modelos de maturidade propõem a aplicação de boas práticas que auxiliam o planejamento, implementação, testes e monitoramento de processos e produtos de uma organização [Tonini et al., 2008]. Desta forma, os modelos de maturidade podem ser usados como guia para o aprimoramento de processos com intuito de fornecer ao cliente uma excelência em qualidade do produto software. Observase que a qualidade do produto deve ser analisada em separado da qualidade do seu processo de construção. De acordo com a norma ISO/IEC 25010 a qualidade do produto software é a sua capacidade de satisfazer as necessidades implícitas e explícitas quando é 
utilizado em condições específicas. Contudo, a qualidade do software pode ser influenciada pela qualidade no seu processo de desenvolvimento, o que cria uma relação entre os modelos de maturidade e a qualidade final do produto de software. Nesse sentido, as certificações dos graus de maturidade de uma organização contribuem para ampliar o grau de confiança do consumidor.

Um processo de desenvolvimento de software produz diversos artefatos que também fazem parte do software, por exemplo, os modelos de projeto, codificações e relatórios de teste. Cada artefato produzido pode ser avaliado em termos qualitativos. $\mathrm{O}$ Catálogo de Transparência de Software [ER-PUC-Rio, 2018] é uma ferramenta que visa reunir operacionalizações que instanciem qualidades que permitam a construção de um software mais transparente. A qualidade de transparência é resultante a partir da contribuição de diversas qualidades que compõem o conceito de transparência.

[Sousa et al., 2015] identificou a existência de correlações entre o modelo de Melhoria de Processos do Software Brasileiro (MPS.BR) [Softex, 2018] e o Catálogo de Transparência, identificando a possibilidade de uma contribuição mútua entre as propostas. Isso ocorre devido porque diversas qualidades demandadas por ambas as propostas são similares, o que define uma interseção entre as propostas. Nesse sentido, o catálogo de transparência de software poderia ser utilizado para construir processos de desenvolvimento de software a partir das operacionalizações propostas no catálogo. Entretanto, o trabalho de [Sousa et al., 2015] aprofundou-se apenas no Nível F do MPS.BR, em especial, no processo de Gerência de Configuração (CGO).

Neste trabalho, estendemos essa investigação para o processo Definição do Processo Organizacional (DFP) do nível E do MPS.BR. O procedimento de investigação foi similar ao de [Sousa et al., 2015], se baseando na identificação de conceitos de qualidade (definidos por palavras-chave) presentes em ambas as propostas. Os resultados demonstram a existência da interseção do DFP com as características de Transparência.

\section{Referencial Teórico}

\section{Transparência de Software}

O conceito de Transparência é um assunto constantemente debatido devido a sua importância e a sua valorização cada vez maior por parte de toda a sociedade. A Transparência é um conceito qualitativo, considerado na Engenharia de Software um requisito não funcional. Um dos desafios atuais dos profissionais de tecnologia é a construção de softwares Transparentes [Sousa et al. 2015].

O Grupo de Engenharia de Requisitos da PUC-Rio estuda o conceito de Transparência aplicado a diversos domínios (ex. [Leal et al., 2011], [Engiel\&Leite, 2016], [Portugal\&Leite, 2015]). Um dos produtos do grupo de pesquisa é o Catálogo de Transparência de Software que se propõem a servir de guia para instanciação de qualidades relacionadas à Transparência em Software, bem como uma base de conhecimento para operacionalizações que são capazes de ampliar o grau das determinadas qualidades para as quais são projetadas [Cappelli\&Leite, 2009]. O catálogo é dividido em três níveis, aplicando o padrão GQO (Goal, Question, Operationalization) [Serrano et al., 2011]. No primeiro nível (Goal) encontra-se o Grafo de Transparência que interliga o conceito de Transparência às qualidades que a compõem, ou seja, que são capazes de contribuir positivamente para Transparência. Esse grafo (Figura 1) é modelado 
na linguagem SIG (Softgoal Interdependency Graph), presente no NFR (Non-Functional Requirement) Framework [Chung, 2009]. O Catálogo de Transparência também aplica o padrão de questões (Question patterns) [Serrano et al., 2011] que consiste na definição de perguntas que delineiam elementos capazes de demonstrar contribuições para a determinada qualidade. No nível das operacionalizações (Operationalization) estão as ações que devem ser capazes de responder positivamente às questões quando corretamente implementadas.

A Figura 1 apresenta o grafo de Transparência no formato SIG, detalhando as diversas qualidades relacionadas. O relacionamento "help" representa o tipo de contribuição (positiva).

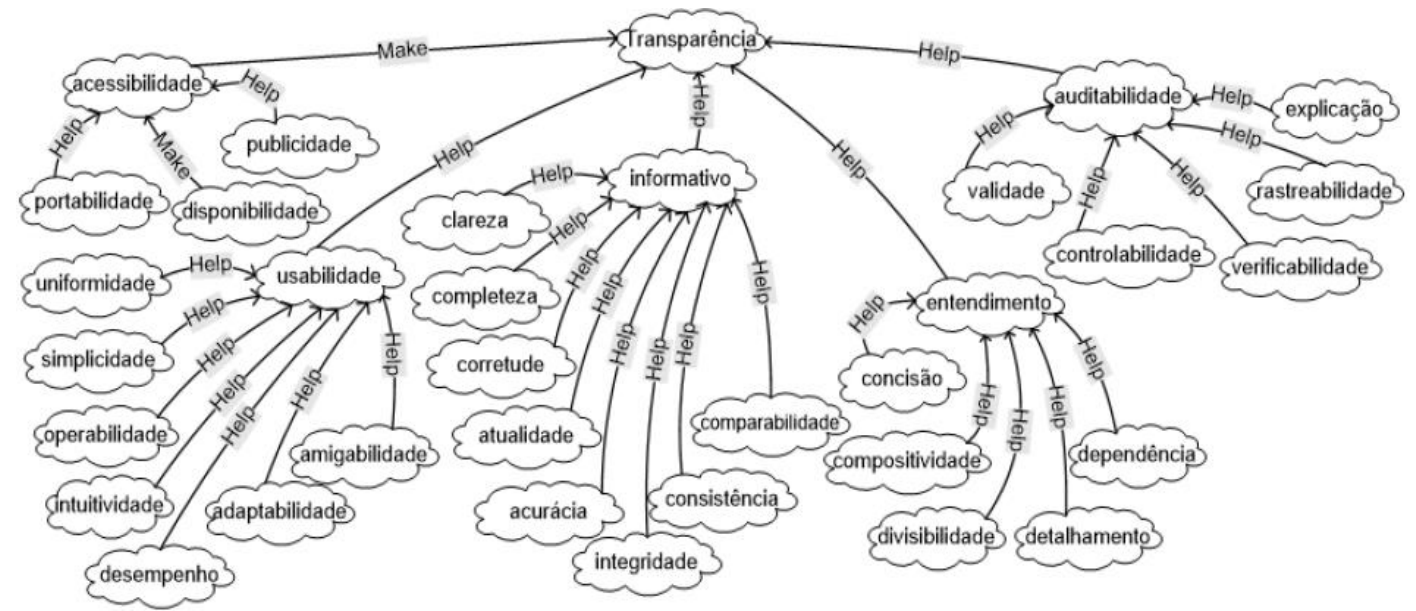

Figura 1. Grafo SIG de Transparência [Cappelli, 2009]

\section{MPS.BR}

O MPS.BR é um programa da [Softex, 2018] que visa a melhoria de processos de desenvolvimento de software, serviços e as práticas de gestão de RH na indústria de TIC. Os níveis de maturidade no modelo MPS.BR estabelecem patamares de evolução dos processos de software. A partir do nível de maturidade de uma organização é possível criar uma expectativa de seu desempenho.

O MPS.BR define sete níveis de maturidade: A (Em Otimização), B (Gerenciado Quantitativamente), C (Definido), D (Largamente Definido), E (Parcialmente Definido), F (Gerenciado) e G (Parcialmente Gerenciado), sendo o nível A o nível máximo que a empresa poderá atingir. Cada nível é composto por um conjunto de processos. Neste trabalho, abordamos especificamente o processo Definição do Processo Organizacional (DFP) que compõem o Nível $\mathrm{E}$, e tem como foco a padronização dos processos da organização.

\section{Mapeando interseções de qualidade entre o processo DFP do Nível E do MPS.BR e da Transparência}

O nível E possui foco na padronização de processos e uso de melhores práticas. É composto pelos processos Avaliação e Melhoria do Processo Organizacional, Definição do Processo Organizacional, Gerência de Recursos Humanos, Gerência de Reutilização e Gerência de Projetos (evolução). O processo DFP é composto por oito Resultados Esperados (RE). Os REs são sentenças que expressam elementos que devem estar 
presentes no processo ou resultar a partir da sua execução. O mapeamento das interseções entre o DFP e Transparência é baseado nos enunciados que descrevem os REs. Esses enunciados explicitam os elementos que devem estar presentes para que a RE seja satisfeita. Na RE, são identificadas palavras-chave que representam conceitos também presentes nas características de Transparência. A identificação de palavras-chave também foi utilizada em [Sousa et al., 2015]. Inicialmente o enunciado do propósito do processo DFP é analisado. As palavras-chave que foram identificadas são marcadas em negrito para destaque e justificadas em sequência: O propósito do processo DFP é estabelecer e manter um conjunto de ativos de processo organizacional e padrões do ambiente de trabalho usáveis e aplicáveis às necessidades de negócio da organização. A partir das palavras-chave, relacionamos algumas características que possuem o potencial de contribuir com o propósito: Usabilidade - visando manter a padronização e que sejam usáveis e aplicáveis; Disponibilidade - visando garantir que os ativos sejam utilizados sempre que necessário; Entendimento - para auxiliar ao usuário no adequado uso e aplicação dor processos; Informativo - para ampliar a qualidade da informação de especificação e uso do processo.

Em seguida apresentamos a avaliação dos enunciados dos REs. As propostas de operacionalização são ilustradas e justificadas para cada RE. As palavras-chave são identificadas em negrito. No primeiro RE as seguintes palavras-chave são identificadas: "Um conjunto definido de processos padrão é estabelecido e mantido, juntamente com a indicação da aplicabilidade de cada processo". A primeira operacionalização proposta é "Definir Método para Criação de Processos". A criação destes processos pode ocorrer a partir de um único processo integrado com todas as atividades que são realizadas para desenvolver ou manter um produto de software; ou pela definição de um processo padrão para cada processo implementado. Neste último, é necessário que se determine a sequência e a interação dos processos padrão com os demais processos. A segunda operacionalização é "Verificar Aplicabilidade de Processos". Trata-se de uma análise do impacto que poderá ocorrer em caso de implantação ou modificação processo. A Adaptabilidade é a característica de Transparência que possui uma forte correlação com a DFP1, visto que ela contribui na capacidade de um processo ser alterado sem impacto na sua aplicabilidade. Foram propostas as operacionalizações: "Utilizar Método para Configuração de Processo"; "Configurar Ferramenta para Verificação de Adaptabilidade"; "Elaborar Catálogo de Processos Definidos" (Figura 2).

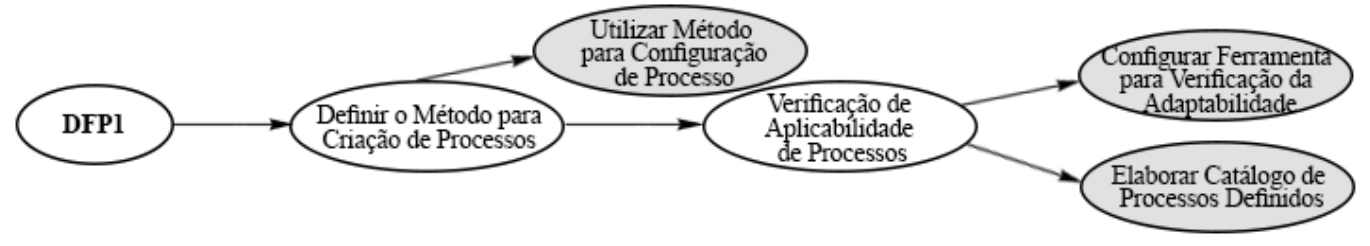

Figura 2. Operacionalização para o Resultado Esperado DFP1

No segundo RE as seguintes palavras-chave são identificadas: "Uma biblioteca de ativos de processo organizacional é estabelecida e mantida.". A operacionalização de "Documentar Ativos de Processos" é recomendada por ser uma atividade executada continuamente durante os projetos da organização a fim de inserir tanto a definição dos processos padrões quanto os ativos de processos, para que assim possam ser mantidos e recuperados a qualquer momento em uma biblioteca, como um sistema de controle de versão, por exemplo. É necessário ainda entender que com a evolução dos processos é 
essencial que haja políticas de acompanhamento a fim de atualizar a biblioteca de ativos. A Controlabilidade possui uma forte relação com a documentação de ativos, sendo assim essa característica de Transparência auxilia na operacionalização da DFP2. Desta forma, as seguintes operacionalizações são propostas: "Criar Ferramenta para Controle de Versão"; "Conceber Políticas de Acompanhamento"; "Classificar Desvios de Processos" (Figura 3).

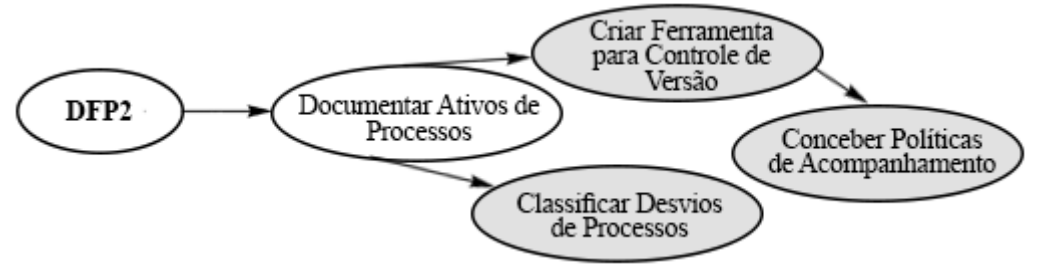

Figura 3. Operacionalização para o Resultado Esperado DFP2

No terceiro RE as seguintes palavras-chave são identificadas: "Tarefas, atividades, papéis e produtos de trabalho associados aos processos padrão são identificados e detalhados, juntamente com o desempenho esperado do processo.". A partir de uma investigação sobre a referida pergunta pode-se sugerir algumas operacionalizações, como a "Definir Informações de Processos", deve-se estabelecer critérios padronizados para a identificação e detalhamento de tarefas, atividades, papéis e produtos de trabalho referente ao processo a ser detalhado. Tal detalhamento pode conter, por exemplo, o nome da atividade, a descrição da mesma, a pré atividade, os critérios de entrada e saída para que a atividade seja iniciada e finalizada, o responsável pela atividade, bem como os participantes, os produtos requeridos, os produtos gerados e as ferramentas que serão utilizadas para a sua execução. Outra operacionalização é a "Analisar Desempenho de Processo", consiste na verificação do impacto que o processo poderá causar. Este desempenho pode ser quantificado pela medição de atributos dos produtos produzidos pelos processos ou por meio da medição direta de atributos dos processos [Florac\&Carleton, 1999]. Ao analisar as características relacionadas com a DFP3 constatou-se que Desempenho tem forte ligação, isso ocorre pois a partir da identificação e detalhamento dos processos é possível estabelecer um melhor desempenho na execução dos processos. Neste caso são propostas as operacionalizações: "Definir Glossário Técnico"; "Padronizar Nomes e Termos”; "Utilizar Método de Comparação de Desempenho" (Figura 4).

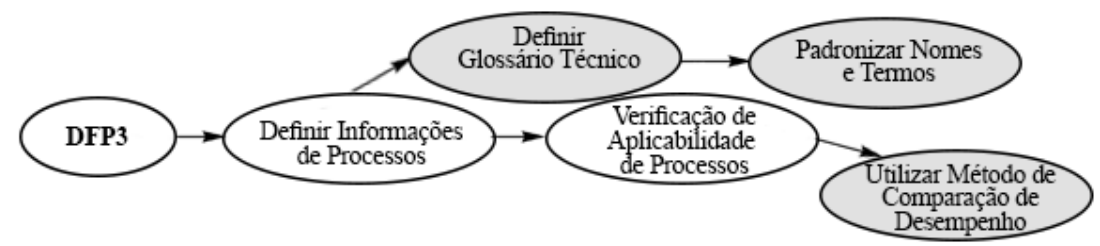

Figura 4. Operacionalização para o Resultado Esperado DFP3

No quarto RE as seguintes palavras-chave são identificadas: "As descrições dos modelos de ciclo de vida a serem utilizados nos projetos da organização são estabelecidas e mantidas.", na Figura 5 temos a operacionalização proposta de "Definir dos Ciclo de Vida de Processos". Existem diversas propostas de ciclos de vida de software que são aplicados a diferentes tipos projetos, por exemplo, cascata, incremental e evolutivo. Com isso devem ser definidos, revisados e atualizado os possíveis ciclos de vida adotados pela organização. Ao se analisar sobre o ponto de vista de transparência constatou-se que é 
necessário a Consistência sobre esse resultado esperado, com isso, foram sugeridas as operacionalizações: "Definir Políticas e Padrões de Modelagem de Processos"; "Comparar Etapas do Processo com Dados Armazenados"; "Comparar Resultados com Objetivos do Processo".

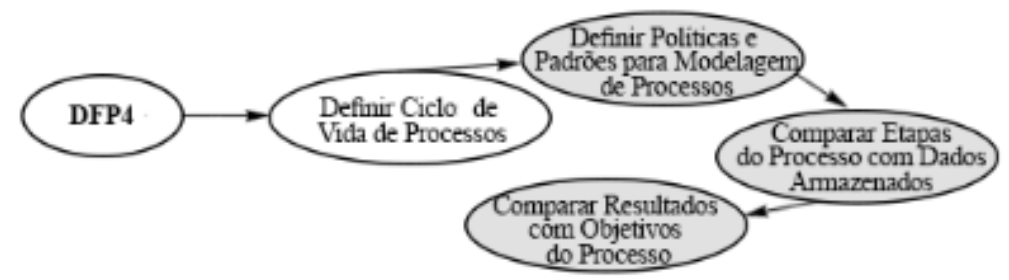

Figura 5. Operacionalização para o Resultado Esperado DFP4

No quinto RE as seguintes palavras-chave são identificadas: "Uma estratégia para adaptação do processo padrão é desenvolvida considerando as necessidades dos projetos.", como operacionalização foi sugerida a utilização da Base de Informações dos Projetos, onde contém as informações a respeito dos projetos realizados pela empresa e assim auxiliar na adaptação, ou pode realizar a operacionalização de Interpretar Processos, neste caso o guia de processos padrões é utilizado para adaptação. Guia de Adaptação de Processos, guia de boas práticas de adaptação de processos baseado nos processos definidos em relação às necessidades identificadas na base de informações atualizadas. Critérios e guias devem ser definidas para que o processo padrão da organização possa atender as necessidades do projeto, os critérios e guias devem ser capazes de descrever, por exemplo, que processo utilizar dentro do conjunto de processos disponíveis, quais atividades são opcionais e quais não são de acordo com as características do projeto, etc. Por envolver questões de adaptação de processos é necessário um alto grau de Controlabilidade, para a qual são propostas as seguintes operacionalizações: "Gerir Controle de Versão"; "Adaptar Processos"; "Acompanhar Demanda de Adaptação de Processos” (Figura 6).

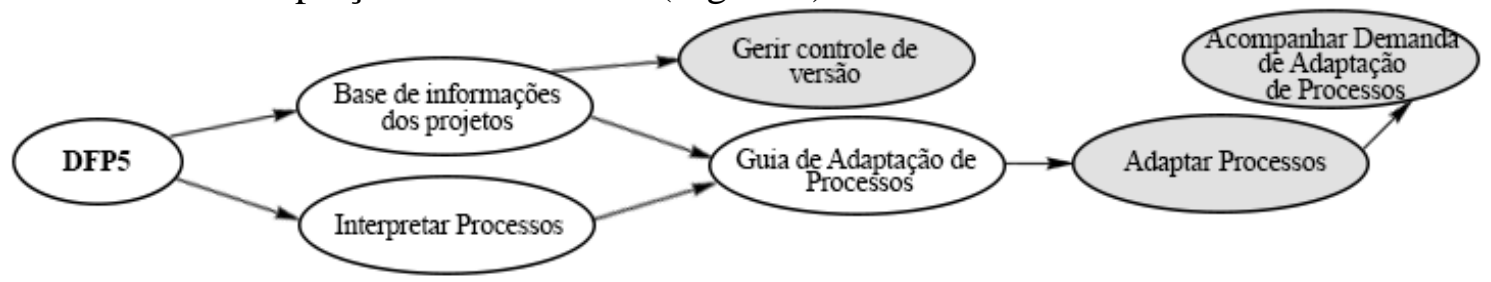

Figura 6. Operacionalização para o Resultado Esperado DFP5

No sexto RE as seguintes palavras-chave são identificadas: O repositório de medidas da organização é estabelecido e mantido. Como operacionalizações foram propostas: "Definir de Medidas para Processos Organizacionais" - deve se incluir em um repositório informações necessárias para entender e interpretar as medidas dos processos e produtos relacionados ao conjunto de processos padrões da organização; "Acompanhar os Processos Organizacionais" - a partir da análise das medidas a fim de aumentar a compreensão sobre os processos, atualizações no repositório podem ser realizadas, com isso é necessário o acompanhamento dos processos organizacionais a fim de avaliar a adequação e aplicabilidade dessas medidas. Foi constatada a necessidade de Detalhamento para a execução das operacionalizações. Para isso são propostas as operacionalizações: "Definir Glossário Técnico"; "Padronizar Nomes e Termos"; "Comparar Resultados com Objetivo do Processo" (Figura 7). 


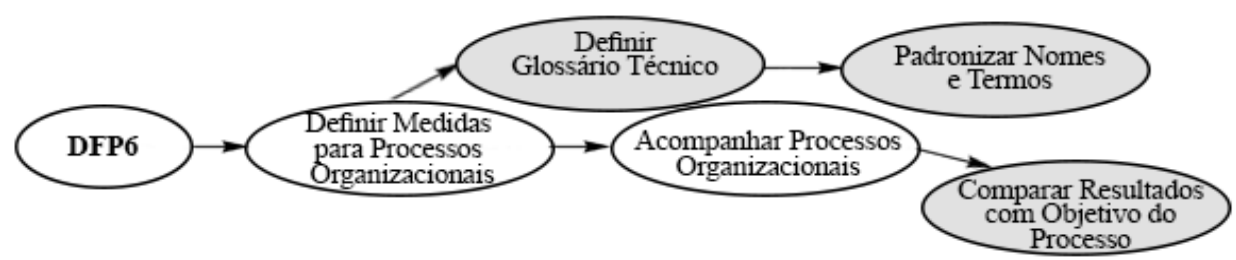

Figura 7. Operacionalização para o Resultado Esperado DFP6

No sétimo RE as seguintes palavras-chave são identificadas: "Os ambientes padrão de trabalho da organização são estabelecidos e mantidos. É sugerida a operacionalização de "Documentar os Ambientes Padrão de Trabalho", para identificar os ambientes definidos como padrão e realidade organizacional. Realizando constante atualização das documentações espelhando a realidade organizacional do momento e seu histórico. Ao analisar as características de Transparência constatou-se que a Completeza é a que possui uma forte relação com esse RE. Portanto, foram propostas as operacionalizações: "Definir Pontos de Vista Abordados"; "Definir Métodos de Validação de Dados"; "Validar Eficácia para os Interessados” (Figura 8).

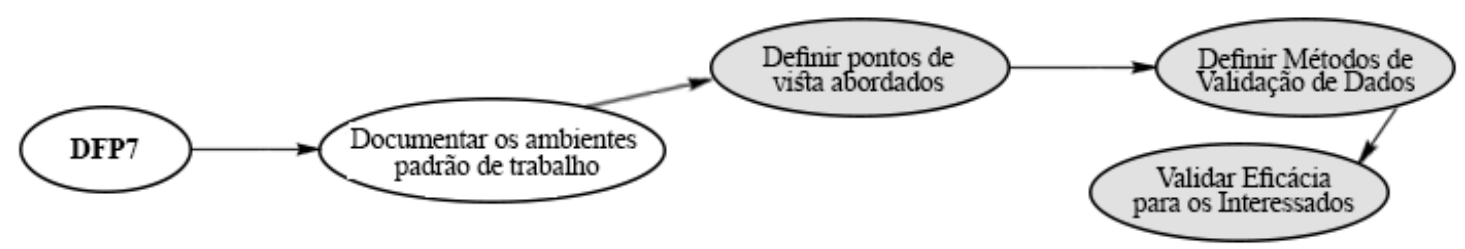

Figura 8. Operacionalização para o Resultado Esperado DFP7

No oitavo RE as seguintes palavras-chave são identificadas: "Regras e diretrizes para a estruturação, formação e atuação de equipes são estabelecidas e mantidas. Sugere-se a operacionalização "Criar Guia de Estrutura Hierárquica de Equipes" identifica os diversos tipos de atividades necessárias na equipe e organizá-las em uma estrutura base que seja facilmente replicável e esteja em constante atualização pela necessidade e realidade do projeto. A qualidade de Compositividade possui uma forte ligação com este RE pois preza pela capacidade de construir a partir de diferentes partes o que se correlaciona com concepção de regras e diretrizes. Sendo assim, foram propostas as operacionalizações "Definir Atividades Necessárias na Equipe"; "Identificar Perfis Adequados para Cada Atividades"; "Formar Equipes" (Figura 9).

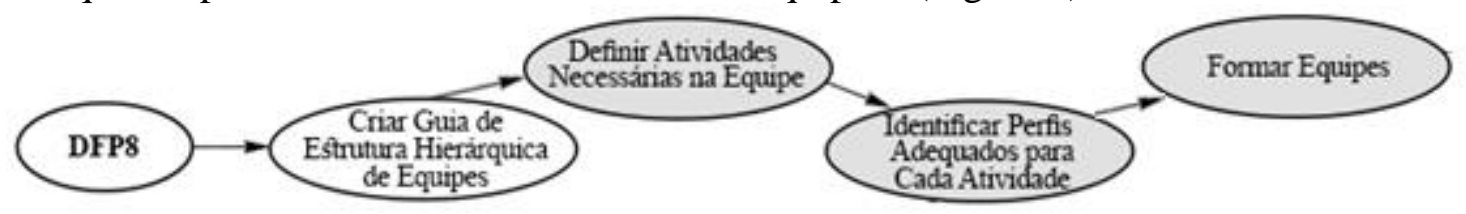

Figura 9. Operacionalização para o Resultado Esperado DFP8

\section{Conclusão}

Este trabalho teve por objetivo verificar as possíveis relações entre o processo DFP e as características de Transparência. A partir de palavras-chave dos enunciados foram identificadas as relações existentes. Posteriormente foram propostas operacionalizações.

Com o resultado desta pesquisa é possível compreender a existência de uma correlação entre as propostas do MPS.BR e a Transparência de software e a partir disso 
propor operacionalizações que podem ser incluídas em um catálogo de Transparência, que armazena o conhecimento das operacionalizações dos requisitos não funcionais.

Como trabalho futuro, o mapeamento criado entre as duas propostas contribuirá para a construção de um catálogo de transparência orientado à maturidade de software. Além disso, será dada continuidade às investigações para identificar possíveis relações de outros níveis e proposições de operacionalizações visando a Transparência. $\mathrm{O}$ amadurecimento deste trabalho permitirá no futuro a composição de processos alinhados ao MPS.BR e Transparência a partir do conhecimento registrado no catálogo a ser construído.

\section{Referências}

Cappelli, C., "Uma Abordagem para Transparência em Processos Organizacionais Utilizando Aspectos". Rio de Janeiro, 328 p. Tese de Doutorado - Departamento de Informática, Pontifícia Universidade Católica do Rio de Janeiro, 2009.

Chung, L., Leite, J.C.S.P., "On Non-Functional Requirements in Software Engineering”, Conceptual Modeling: Foundations and Applications, 363-379, 2009.

Engiel, P.; Leite, J.C.S.P., Tornando a Legislação Transparente para o Engenheiro de Requisitos. In: Workshop de Transparência em Sistemas, 2016, v. IV. p. 1-6.

ER-PUC-Rio, "Catálogo de Transparência”. Disponível em: goo.gl/Y6VRnP. Acesso em: 10 de ago. de 2018.

Florac, W.A., Carleton, A.D., Measuring the Software Process - Statistical Process Control for Software Process Improvement, Addison-Wesley, 1999.

Leal, A.L.C.; Sousa, H. P.; Leite, J.C.S.P.; Braga, J.L., Transparência Aplicada a Modelos de Negócio. In: Workshop em Engenharia de Requisitos, p.321-332, 2011.

Portugal, R.; Leite, J.C.S.P., A Transparência do GitHub para uso de Artefatos como fontes de informação na Engenharia de Requisitos, WTRANS'15. v.3.p.1-6, 2015.

Serrano, M., Leite, J.C.S.P., "Capturing Transparency-Related Requirements Patterns through Argumentation", First International Workshop on Requirements Patterns (RePa'11), Trento, Italy, 2011.

SOFTEX, "Rede de Apoio à Indústria Brasileira de Software e Serviços de TI". Disponível em: www.softex.br. Acesso em: 18 ago. 2018.

Sousa, H.P.S, Leal, A.L.C.; Leite, J.C.S.P. "Transparência no contexto do MPS.BR: explorando o nível $\mathrm{F}$ através do alinhamento de características de qualidade" Revista Brasileira de Sistemas de Informação, vol. 8, No. 4, pp. 109-141, 2015.

Tonini, A.C.; De Carvalho, M.M.; De Mesquita, S.M., "Contribuição dos modelos de qualidade e maturidade na melhoria dos processos de software." Production, v. 18, n. 2, p. 275-286, 2008. 